OPEN ACCESS

Edited by:

Peter Sörös,

University of Western Ontario,

Germany

Reviewed by:

Vaughan G. Macefield,

Western Sydney University, Australia

Jon David Levine,

University of California,

San Francisco, USA

*Correspondence:

Sophie L. Wilcox

sophie.wilcox@childrens.harvard.edu

Received: 26 April 2016 Accepted: 08 July 2016

Published: 26 July 2016

Citation:

Wilcox SL, Veggeberg R, Lemme J, Hodkinson DJ, Scrivani S,

Burstein $R$, Becerra $L$ and

Borsook D (2016) Increased Functional Activation of Limbic Brain Regions during Negative Emotional Processing in Migraine.

Front. Hum. Neurosci. 10:366. doi: 10.3389/fnhum.2016.00366

\section{Increased Functional Activation of Limbic Brain Regions during Negative Emotional Processing in Migraine}

\author{
Sophie L. Wilcox ${ }^{1,2 *}$, Rosanna Veggeberg ${ }^{1,3}$, Jordan Lemme ${ }^{2}$, Duncan J. Hodkinson ${ }^{1,2}$, \\ Steven Scrivani ${ }^{4}$, Rami Burstein ${ }^{5}$, Lino Becerra ${ }^{1,2,3}$ and David Borsook $k^{1,2,3}$ \\ ${ }^{1}$ Center for Pain and the Brain (PAIN Research Group), Boston Children's Hospital, Harvard Medical School, Boston, MA, \\ USA, ${ }^{2}$ Department of Anesthesiology, Perioperative and Pain Medicine, Boston Children's Hospital, Harvard Medical \\ School, Boston, MA, USA, ${ }^{3}$ Department of Psychiatry, PAIN Research Group, Brain Imaging Center, McLean Hospital, \\ Harvard Medical School, Belmont, MA, USA, ${ }^{4}$ Department of Oral and Maxillofacial Surgery, Massachusetts General \\ Hospital, Boston, MA, USA, ${ }^{5}$ Anesthesia, Critical Care and Pain Medicine, Beth Israel Deaconess Medical Center, Harvard \\ Medical School, Boston, MA, USA
}

Pain is both an unpleasant sensory and emotional experience. This is highly relevant in migraine where cortical hyperexcitability in response to sensory stimuli (including pain, light, and sound) has been extensively reported. However, migraine may feature a more general enhanced response to aversive stimuli rather than being sensoryspecific. To this end we used functional magnetic resonance imaging to assess neural activation in migraineurs interictaly in response to emotional visual stimuli from the International Affective Picture System. Migraineurs, compared to healthy controls, demonstrated increased neural activity in response to negative emotional stimuli. Most notably in regions overlapping in their involvement in both nociceptive and emotional processing including the posterior cingulate, caudate, amygdala, and thalamus (cluster corrected, $p<0.01$ ). In contrast, migraineurs and healthy controls displayed no and minimal differences in response to positive and neutral emotional stimuli, respectively. These findings support the notion that migraine may feature more generalized altered cerebral processing of aversive/negative stimuli, rather than exclusively to sensory stimuli. A generalized hypersensitivity to aversive stimuli may be an inherent feature of migraine, or a consequential alteration developed over the duration of the disease. This proposed cortical-limbic hypersensitivity may form an important part of the migraine pathophysiology, including psychological comorbidity, and may represent an innate sensitivity to aversive stimuli that underpins attack triggers, attack persistence and (potentially) gradual headache chronification.

Keywords: migraine, headache, fMRI, emotion, IAPS, limbic

Abbreviations: EV, explanatory variable; fMRI, functional magnetic resonance imaging; IAPS, International Affective Picture System; PCC, posterior cingulate cortex; SAM, Self-assessment manikin. 


\section{INTRODUCTION}

Pain, by its definition, is comprised of both "an unpleasant sensory and emotional experience" (Merskey and Bogduk, 1994). This is evident in pain conditions, such as migraine, where emotional factors and headache symptoms have been demonstrated to have a complex, bidirectional relationship (Spierings et al., 1997; Lanteri-Minet et al., 2005; Walters et al., 2014). There is a higher co-occurrence of emotional disturbances including anxiety, depression, phobias and panic disorders in migraineurs, compared to the general population (Radat and Swendsen, 2005). Furthermore, heightened emotion (in particular stress) is the most commonly reported trigger for migraine attacks (Kelman, 2007).

The relationship between pain and emotion is also highlighted by the overlap in their central representation in the brain. Pain (Peyron et al., 2000; Duerden and Albanese, 2013) and emotion (Phan et al., 2002) both activate a wide array of cortical and subcortical regions, overlapping in areas including the insula, cingulate, thalamus, amygdala, and caudate (Vogt, 2005; Cauda et al., 2012). This neuroanatomical overlap is highly relevant given that one pathophysiologic mechanism of migraine is an altered cerebral processing of sensory stimuli (including pain (Burstein et al., 2010; Moulton et al., 2011), light (Boulloche et al., 2010; Bjork et al., 2011; Denuelle et al., 2011; Martin et al., 2011; Cucchiara et al., 2015) and smell (Demarquay et al., 2008; Stankewitz and May, 2011)) attributed to enhanced cortical excitability and/or dishabituation (Coppola et al., 2007; Brighina et al., 2009; Goadsby et al., 2009). However, as previously mentioned, pain is essentially a negative emotional experience. In the context of migraine, where patients may report abnormal sensory tolerances to light (photophobia) (Vanagaite et al., 1997; Mulleners et al., 2001), sound (phonophobia) (Rojahn and Gerhards, 1986; Vingen et al., 1998; Ashkenazi et al., 2009) and smell (Demarquay et al., 2006), these sensory stimuli may also have a significant negative emotional component (Rojahn and Gerhards, 1986). This raises the potential that migraine may feature altered cerebral processing of aversive stimuli in general, rather than sensory specific.

In support of this view a limited number of studies have shown altered processing of emotional stimuli in migraine, specifically in regards to negative or aversive emotion. Andreatta et al. (2012) showed that migraineurs respond to angry, but not neutral or happy, facial expressions preferentially and more intensively than healthy individuals. In relation, de Tommaso et al. (2009) showed that affective images are able to modulate pain perception and cortical response in migraineurs, whereas other modalities of distraction (i.e., mental arithmetic) were not effective.

The purpose of the present study was to assess the brain correlates of emotional processing in patients with episodic migraine during the interictal period using functional Magnetic Resonance Imaging (fMRI). We hypothesized we would observe enhanced neural activation in migraineurs in areas involved in emotional and pain processing (i.e., amygdala, hippocampus, prefrontal cortex, anterior cingulate gyrus) in response to viewing negative pictures. In contrast, we expected no differences between migraineurs and controls in neuronal response to positive and neutral emotional stimuli.

\section{MATERIALS AND METHODS}

\section{Subjects}

The data from forty-six age- and gender-matched subjects ( $n=23$ migraine patients and $n=23$ healthy control subjects) who underwent an imaging session at McLean Hospital were included in this study. All migraine patients fulfilled the International Classification for Headache II criteria for episodic migraine (confirmed by a neurologist) and, as part of the inclusion criteria, suffered from migraines for more than 3 years. Clinical characteristics (including medication usage) for individual migraine subjects are given in Supplementary Table S1. Migraine patients were scanned during their interictal (headache-free) period, defined as migraine free at least $48 \mathrm{~h}$ before and $24 \mathrm{~h}$ after the imaging session. Healthy control subjects had no significant history of migraine, or other headache condition. All subjects were screened for depression [Beck Depression Inventory II (Beck et al., 1996)], exclusionary score $>25$ (moderate to severe depression) and had no history of any other chronic pain, psychiatric (including clinical depression and/or anxiety) or neurological disorder, or any other major disease. Subjects were also screened by a urine test for barbiturates, benzodiazepines, amphetamine, cocaine, tetrahydrocannabinol, phencyclidine, and opioids (excluding prescription pain medications). The study protocol was approved by the Institutional Review Board at McLean Hospital and was conducted in accordance with the principles of the Declaration of Helsinki. Informed consent was obtained from all subjects prior to participation in the study.

\section{Emotional Stimuli and Scanning Paradigm}

Emotional visual stimuli consisted of positive (pleasant), neutral and negative (unpleasant) color photographs selected from the International Affective Picture System (IAPS; Lang et al., 2008). The IAPS is a standardized, emotionally evocative visual stimulus that has been widely used in studies of emotion, including functional imaging (e.g., de Tommaso et al., 2009; Kamping et al., 2013; Neta et al., 2013; Boucher et al., 2015). A total of 100 IAPS images were displayed ( 30 positive, 30 negative, and 40 neutral images). The baseline visual stimuli consisted of a black cross hair presented in the middle of a gray screen, a control condition used in other fMRI studies of emotion (Gur et al., 2002; Aldhafeeri et al., 2012). The experimental paradigm presented in the scanner consisted of 10 blocks of emotional visual stimuli with duration for each block of $25 \mathrm{~s}$ followed by the baseline visual stimuli with duration of $30 \mathrm{~s}$, with the exception of the first and last baseline with duration of $1 \mathrm{~min}$. During each emotional visual block 10 IAPS images were presented for $2.5 \mathrm{~s}$ each. Subjects were not aware of the presentation order and emotional-visual blocks were presented in the same pseudo-randomized order for all subjects (Figure 1). The timing of the visual stimuli was programmed using Microsoft PowerPoint. Subjects lay in the MRI scanner 


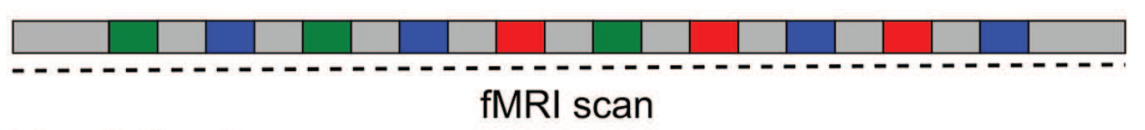

Visual stimuli:

positive

neutral

negative

baseline

FIGURE 1 | Schematic diagram of the experimental paradigm during the fMRI scan. Visual stimuli (International Affective Picture System) blocks were presented in a pseudo-randomized order.

and viewed, via a mirror affixed to the head coil, visual stimuli projected onto a screen placed behind the scanner. Before the paradigm began subjects were instructed to keep their eyes open throughout the scan, to blink as normal and to fixate on the cross hair during the baseline visual stimuli. After the imaging session subjects were reshown the presentation and asked to rate each stimulus block for their average emotional valence and arousal using the Self-assessment manikin (SAM) scale (Bradley and Lang, 1994). The SAM scale is an affective rating system that uses graphic figures (manikins) to depict emotional reactions. Subjects were instructed to select any of the nine sequential figures comprising each scale, which resulted in a 9-point rating scale for valence and arousal. Ratings were scored such that a higher score represents a high rating on each scale.

\section{fMRI Data Acquisition}

MRI data were collected on a 3 Tesla Siemens Trio scanner with a 12-channel phased array head coil (Erlangen, Germany). fMRI data were collected using a gradient echo-echo planar pulse sequence with $3.5 \mathrm{~mm} \times 3.5 \mathrm{~mm} \times 3.5 \mathrm{~mm}$ resolution. fMRI scan parameters: time of Repetition $=2500 \mathrm{~ms}$, Time of Echo $=30 \mathrm{~ms}$, Field of View $=224 \times 224$, Flip Angle $=90^{\circ}$, \# of Slices $=41$ axial slices, \# of Volumes $=256$. MagnetizationPrepared Rapid Acquisition Gradient-Echo anatomical images were also collected. Anatomical scan parameters: time of Repetition $=2000 \mathrm{~ms}$, Time of Echo $=3.53 \mathrm{~ms}$, Time of Inversion $=1100 \mathrm{~ms}$, Flip Angle $=8^{\circ}, 224$ sagittal slices.

\section{fMRI Data Preprocessing and Analysis}

Functional magnetic resonance imaging data processing and analysis was carried out using FEAT (FMRI Expert Analysis Tool) Version 6.00, part of FSL (FMRIB's Software Library ${ }^{1}$ ). Pre-statistics processing included; motion correction using the Linear Motion Correction tool (MCFLIRT); slice-timing correction using Fourier-space time-series phase-shifting for interleaved slice acquisition; non-brain removal using the Brain Extraction tool (BET); spatial smoothing of FWHM $5 \mathrm{~mm}$; grand-mean intensity normalization of the entire 4D dataset by a single multiplicative factor; highpass temporal filtering with a $80 \mathrm{~s}$ cutoff. No volumes were deleted as three dummy scans were acquired and discarded during acquisition to allow for signal equilibration. Functional images were registered to highresolution structural images, which in turn were registered to

\footnotetext{
${ }^{1}$ www.fmrib.ox.ac.uk/fsl
}

standard space (MNI152 average image) using the Linear Image Registration tool (FLIRT).

First level/time-series statistical analysis of single subject data was carried out using FMRIB's Improved Linear Modeling (FILM) with local autocorrelation correction. Three sets of explanatory variables (EVs) were generated based on boxcar functions that corresponded with the visual presentation of the negative, neutral, and positive stimulus blocks. EVs were convoluted with a gamma hemodynamic response function. Standard motion parameters (as estimated by MCFLIRT) were included in the model as confound EVs. $Z$ (Gaussianised T/F) statistic images were thresholded using clusters determined by $Z>2.3$ and a (corrected) cluster significance threshold of $p<0.05$

Higher-level analysis was carried out using FLAME (FMRIB's Local Analysis of Mixed Effects). A mixed effects contrast analysis was performed to compare migraine versus control group activation for each emotion category. $Z$ (Gaussianised T/F) statistic images were thresholded using clusters determined by $z>2.3$ and a (corrected) cluster significance threshold of $p<0.01$ (Worsley, 2001). Significant clusters and their local maxima were identified anatomically using the Harvard-Oxford cortical and subcortical structural atlases (Desikan et al., 2006).

\section{International Affective Picture System (IAPS) Rating Statistical Analysis}

As aforementioned, after the imaging session subjects were reshown the presentation and asked to rate each stimulus block for emotional valence and arousal using the 9-point SAM scale. Each subject's rating of valence and arousal for each stimulus block were averaged for each emotional type (i.e., positive, neutral, and negative). The mean valence and arousal ratings for each emotional type were compared between migraine patients and controls by an independent sample $t$-test, with $p<0.05$ taken as statistically significant.

\section{RESULTS}

\section{Subject Demographics and IAPS Valence and Arousal Ratings}

Migraine subjects consisted of 20 females and 3 males, with an average age of $32.9 \pm 10.0$ years. Controls subjects consisted of 19 females and 4 males, with an average age of $31.3 \pm 9.3$. Migraine and control subjects did not differ in mean age or group 


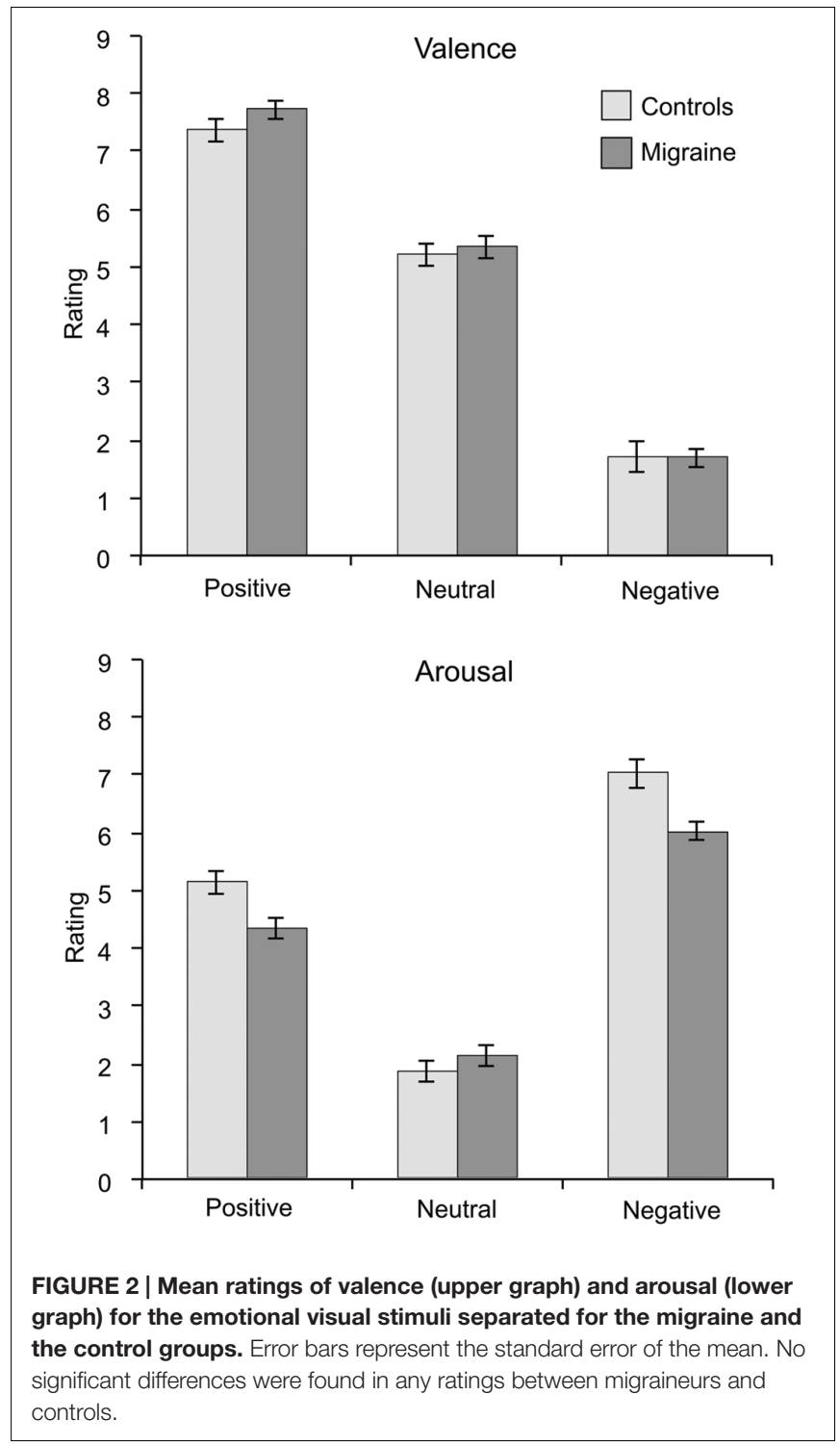

gender composition. Migraineurs averaged $5.4 \pm 4.1$ headaches per month and they had an average duration of the condition of $15.4 \pm 9.2$ years.

Migraine and control subjects did not differ in their ratings of valence or arousal in any emotional category [Positive valence: $t(44)=1.415, p=0.164$; Positive arousal: $t(44)=-1.497$, $p=0.142$; Neutral valence: $t(44)=0.487, p=0.629$; Neutral arousal: $t(44)=0.826, p=0.413$; Negative valence: $t(44)=-$ 0.044, $p=0.965$; Negative arousal: $t(44)=-1.454, p=0.153$ ]. Mean (and standard error of the mean) ratings for migraine and control groups are shown in Figure 2.

\section{Neural Activation during Emotional Stimuli}

Separately, both migraine and control subjects showed similar networks of activated brain regions in response to emotional stimuli (See Supplementary Figure S1).
Positive IAPS stimuli: during positive emotional stimuli migraineurs displayed no significant different in neural response, in comparison to controls (Table 1; Figure 3 [Left panel]).

Neutral IAPS stimuli: during neutral emotional stimuli migraineurs displayed only a single region of increased neural response, in comparison to controls (Table 1; Figure 3 [Middle panel]). This increased activation was observed in visual areas, notably the intracalcarine cortex and lingual gyrus.

Negative IAPS stimuli: during negative emotional stimuli migraineurs displayed a range of regions with increased neural response, in comparison to controls (Table 1; Figure 3 [Right panel] and Figure 4). These included increased activation in the superior and middle frontal gyrus, the frontal medial cortex, the frontal pole, posterior cingulate gyrus, precuneus and cuneal cortex, caudate, thalamus, left amygdala and right hippocampus, brainstem (midbrain and pons) and the cerebellum.

\section{DISCUSSION}

This study investigated, for the first time, brain activation associated with emotional processing in episodic migraine during their interictal period. As hypothesized, an increase in neural activity in migraineurs in response to negative IAPS pictures was observed, notably in regions overlapping in their involvement in both nociceptive and emotional processing including the posterior cingulate, caudate, amygdala, and thalamus. These findings support the notion that migraine may feature more generalized altered cerebral processing of aversive/negative stimuli, rather than restricted to sensory stimuli specifically. This more generalized alteration may be an inherent feature of migraine, or may be a consequential alteration produced by the ongoing and repeated nature of migraine. Regardless of its sequela, this more generalized cortical and subcortical increased functional response may form an important part of the migraine pathophysiology and represent an innate sensitivity to aversive stimuli that underpins attack triggers, attack persistence and potentially chronification (Burstein et al., 2015).

\section{Emotional Appraisal in Migraine}

The categories of emotional (positive, neutral, and negative) compared in this study were induced using IAPS (Lang et al., 2008), a standardized affective picture set which has been used extensively in functional neuroimaging studies of emotional processing (de Tommaso et al., 2009; Kamping et al., 2013; Neta et al., 2013; Boucher et al., 2015). Interestingly, ratings of valence and arousal for all three emotional categories did not differ between migraine subjects and healthy controls. In this regard, the increased neural activity in negative emotional processing in migraineurs was not driven by an enhanced conscious emotional impact of the IAPS stimuli. Steppacher et al. (2015) also reported a similar lack of difference in ratings, while in contrast de Tommaso et al. (2009) reported enhanced emotional impact in migraineurs for both positive and negative IAPS stimuli while in concurrent laser-generated pain. In contrast both Steppacher's and our study were conducted 
TABLE 1 | Regions of significantly different neural activation in migraineurs, compared to healthy controls, during viewing of emotional stimuli.

\begin{tabular}{|c|c|c|c|c|c|c|c|}
\hline \multirow[t]{2}{*}{ Brain region } & \multirow[t]{2}{*}{ Laterality } & \multirow[t]{2}{*}{ Z-stat } & \multirow[t]{2}{*}{$P$} & \multicolumn{3}{|c|}{ MNI coordinates } & \multirow[t]{2}{*}{ Voxels } \\
\hline & & & & $X(\mathrm{~mm})$ & $Y(\mathrm{~mm})$ & $Z(\mathrm{~mm})$ & \\
\hline \multicolumn{8}{|l|}{ Positive emotional stimuli: } \\
\hline \multicolumn{8}{|l|}{ N.A. } \\
\hline \multicolumn{8}{|l|}{ Neutral emotional stimuli: } \\
\hline \multicolumn{8}{|l|}{ Migraine $>$ Controls } \\
\hline Lingual gyrus & $R$ & 3.63 & 0.002 & 4 & -74 & -10 & 550 \\
\hline \multicolumn{8}{|l|}{ Additional Local Maxima } \\
\hline Lingual gyrus & $L$ & 3.48 & & -4 & -66 & 0 & \\
\hline Intracalcarine cortex & $\mathrm{R}$ & 3.46 & & 8 & -86 & 2 & \\
\hline Lingual gyrus & $\mathrm{R}$ & 3.34 & & 14 & -76 & -6 & \\
\hline Lingual gyrus & $R$ & 3.30 & & 24 & -70 & 0 & \\
\hline Lingual gyrus & $L$ & 3.19 & & -2 & -68 & 4 & \\
\hline \multicolumn{8}{|l|}{ Negative emotional stimuli: } \\
\hline \multicolumn{8}{|l|}{ Migraine $>$ Controls } \\
\hline Precuneus cortex & $L$ & 5.1 & $3.23 e^{-26}$ & -12 & -60 & 50 & 7919 \\
\hline \multicolumn{8}{|l|}{ Additional local maxima } \\
\hline Cuneal cortex & $L$ & 5.08 & & 0 & -88 & 32 & \\
\hline Thalamus (Medial dorsal nucleus) & $L$ & 4.69 & & -4 & -14 & 6 & \\
\hline Lingual gyrus & $\mathrm{L}$ & 4.5 & & -2 & -64 & 2 & \\
\hline Precuneus cortex & $L$ & 4.45 & & -14 & -60 & 46 & \\
\hline Precuneus cortex & $\mathrm{R}$ & 4.31 & & 10 & -52 & 4 & \\
\hline Frontal Medial cortex & $\mathrm{R}$ & 3.83 & $1.13 e^{-06}$ & 2 & 50 & -22 & 1228 \\
\hline \multicolumn{8}{|l|}{ Additional local maxima } \\
\hline Frontal medial cortex/Anterior cingulate & $\mathrm{R}$ & 3.78 & & 12 & 34 & -14 & \\
\hline Frontal pole & $L$ & 3.71 & & -14 & 42 & -24 & \\
\hline Frontal medial cortex & $L$ & 3.61 & & -12 & 46 & -14 & \\
\hline Frontal pole & $\mathrm{R}$ & 3.58 & & 8 & 56 & -4 & \\
\hline Frontal medial cortex & $\mathrm{R}$ & 3.52 & & 4 & 54 & -10 & \\
\hline Frontal pole & $\mathrm{R}$ & 4.63 & $3.73 e^{-05}$ & 22 & 38 & 40 & 895 \\
\hline \multicolumn{8}{|l|}{ Additional local maxima } \\
\hline Superior frontal gyrus & $\mathrm{R}$ & 3.57 & & 22 & 30 & 38 & \\
\hline Frontal pole & $\mathrm{R}$ & 3.56 & & 16 & 40 & 40 & \\
\hline Precentral gyrus & $\mathrm{R}$ & 3.35 & & 38 & 2 & 40 & \\
\hline Superior frontal gyrus & $\mathrm{R}$ & 3.28 & & 26 & 10 & 68 & \\
\hline Middle frontal gyrus & $\mathrm{R}$ & 3.23 & & 40 & 6 & 40 & \\
\hline Middle frontal gyrus & $L$ & 4.19 & $1.48 e^{-04}$ & -30 & 22 & 54 & 773 \\
\hline \multicolumn{8}{|l|}{ Additional local maxima } \\
\hline Middle frontal gyrus & $L$ & 4.11 & & -30 & 18 & 56 & \\
\hline Middle frontal gyrus & $L$ & 3.69 & & -52 & 12 & 44 & \\
\hline Middle frontal gyrus & $\mathrm{L}$ & 3.51 & & -48 & 14 & 40 & \\
\hline Middle frontal gyrus & $L$ & 3.38 & & -42 & 4 & 56 & \\
\hline Middle frontal gyrus & $\mathrm{L}$ & 3.29 & & -26 & 32 & 36 & \\
\hline
\end{tabular}

during the interictal (pain-free) state. Taken together this raises the potential that conscious emotional appraisal in migraineurs may be significantly impacted only during a painful state. In support of this theory diary studies of psychological variation in migraineurs over time have shown increased selfreport of stress and negative mood directly proceeding and during headache attacks (Harrigan et al., 1984; Sorbi et al., 1996; Spierings et al., 1996). Further study directly contrasting emotional appraisal in the pain and pain-free phases in migraine are needed to clarify this difference and may indeed demonstrate phase-dependent perception and processing of emotional stimuli.

\section{Central Representation of Pain and Negative Affect}

By definition, pain includes both a sensory and affective component (Merskey, 1968; Merskey and Bogduk, 1994). Nociceptive stimuli, including those of trigeminovascular origin involved in headache, activate a wide array of cortical and subcortical areas (Peyron et al., 2000; DaSilva et al., 2002; 


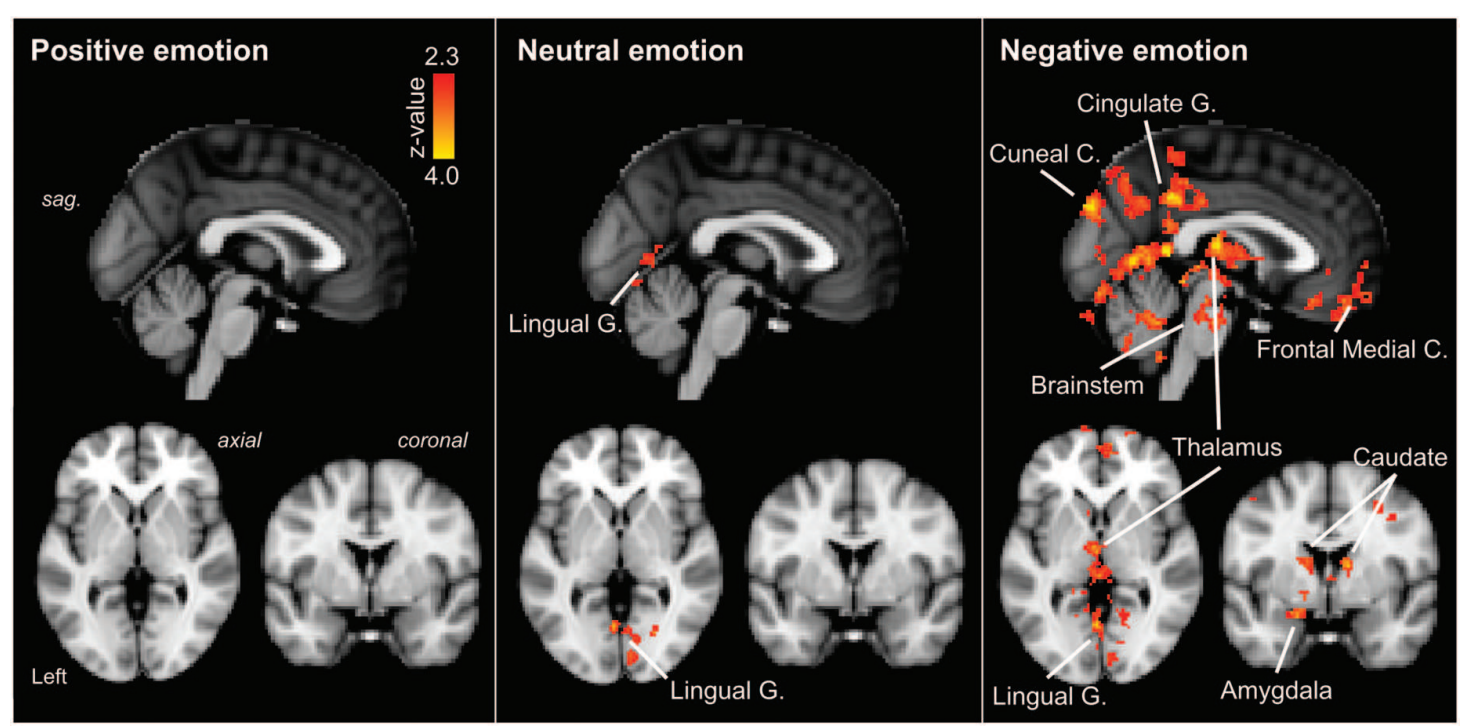

FIGURE 3 | Regions of significantly increased neural activation in migraineurs, compared to healthy controls, in response to emotional stimuli. Left panel: positive emotional stimuli, Middle panel: neutral emotional stimuli, Right panel: negative emotional stimuli. The red-yellow color scale represents $Z$ values for the contrast of migraineurs greater than controls. Significant clusters are overlaid onto the average MNI152 T1-weighted anatomical brain template. G. - Gyrus, C. - Cortex, sag. - sagittal.

Apkarian et al., 2005). How activity in these regions gives rise to the complex experience of pain, including both sensory and affective components, remains an area of active discussion (Melzack, 1999; Iannetti and Mouraux, 2010). These areas involved in pain processing share significant spatial overlap with areas involved in processing other domains including emotion, interoception, and reward (Cauda et al., 2012). Interestingly, these areas of overlap were among the regions of increased activity in response to negative emotional stimuli in migraineurs, notably including limbic structures such as the posterior cingulate, amygdala, hippocampus and the (medial dorsal) thalamus (Figure 4).

However, the region of increased activation in the cingulate region was ascribed to the posterior cingulate cortex (PCC), which is thought to have little or no involvement in pain processing (in regards to acute pain in healthy subjects). Rather the posterior cingulate has extensive connections with the parietal lobe (which also displayed regions of increased activation) and is proposed to be involved in emotional evaluation including assessment of the self-relevance of emotional stimuli (Vogt, 2005). While the PCC may not be involved in processing physical pain, evidence suggests its involvement in processing psychological pain (including grief and sadness; Meerwijk et al., 2013). Vogt has posited that inactivation of the PCC may be one inherit mechanisms by which the emotional component of pain may be reduced (Vogt, 2005). This raises the possibility that in migraineurs the posterior cingulate may be activated (or insufficiently deactivated) in response to nociceptive input (Aderjan et al., 2010; Maleki et al., 2012b; Russo et al., 2012), with the perceptual outcome a more enhanced or maintained pain perception (Shyu and Vogt, 2009).
The amygdala has long been known for its important role in processing the emotional dimension of pain (Gao et al., 2004; Wiech and Tracey, 2009; Neugebauer, 2015). Among its roles it is thought to modulate motor readiness, autonomic functions, and cognitive processes including attention and memory (Zald, 2003). In the context of pain, the connection between the amygdala and sensory cortical regions (Wiech et al., 2014; Bienvenu et al., 2015) allows emotional arousal to facilitate attention and perception. In the long-term, amygdala-driven somatosensory plasticity (increased cortical representation) has been demonstrated in sensory (auditory) processing (Chavez et al., 2009), suggesting that emotional arousal conjoined with a sensory stimulus may, over time, produce an attentional bias, and/or shift in cortical processing (Wiech and Tracey, 2009). In support of the view that amygdala-sensory plasticity may be an important factor in migraine, several studies have demonstrated altered amygdala connectivity with sensory areas (Hadjikhani et al., 2013; Schwedt et al., 2013), however, this has not been a consensus finding (Hougaard et al., 2015).

\section{Migraine Models: Expanding Cortical Hyperexcitability and Chronification}

Our understanding of migraine pathophysiology has dramatically evolved over the last half-century in increasing acknowledgment that this complex neurological disorder affects multiple cortical, subcortical and brainstem regions that regulate sensory, autonomic, cognitive and affective functions (Burstein et al., 2015). The majority of models of migraine mechanisms have focused on features of the migraine attack itself, including peripheral and central sensitization, cortical hyperexcitability, and cortical spreading. Yet, a complete model of migraine must also account for the more complex emotional milieu of 


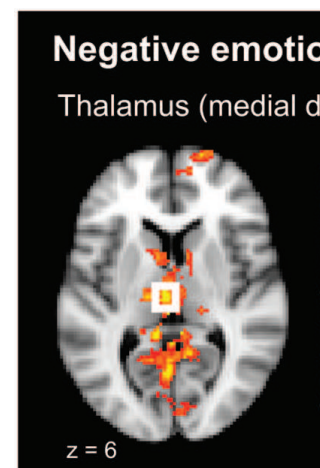

Right hippocampus

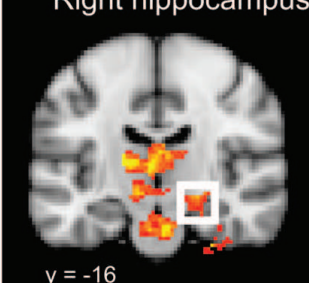

$y=-16$

Left amygdala

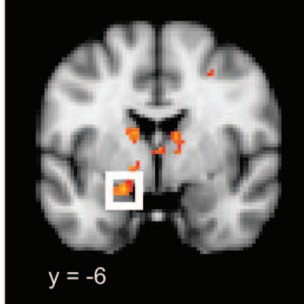

Posterior cingulate

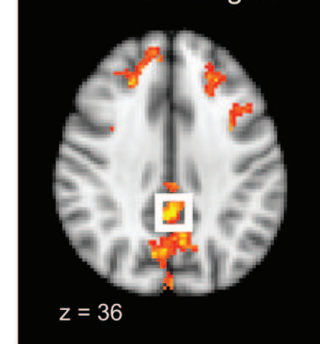

FIGURE 4 | Limbic regions of significantly increased activation in migraineurs, compared to healthy controls, in response to negative emotional stimuli. Limbic regions included the medial dorsal thalamus, amygdala, hippocampus, and posterior cingulate. For each region bar graphs of the mean contrast of parameter estimates (COPE; as a percentage) are presented. Error bars represent the standard error of the mean.

migraine including the prominent role of stress and emotions as attack triggers, prodromal emotional changes, and psychiatric comorbidity. To this end, Maizels et al. (2012) proposed an expansion of the central sensitization model of migraine to include limbic dysfunctions as well as cortical hyperexcitability, a proposal our findings support. This sensitized corticolimbic state would account for the dynamic bidirectional influence of pain and emotion. Limbic regions are also integrated into Burstein and Jakubowski's (2005) "unitary hypothesis" of migraine triggers and exacerbation, which focuses on limbic and hypothalamic regions. Activity in these regions is influenced by common migraine triggers, including stress and emotional responses, and form part of a parasympathetic pathway for the activation of meningeal nociceptors. These same regions also receive extensive projections from the trigeminovascular nociceptive pathway and are placed to initiate symptoms commonly triggered by headache including stress, fatigue, nausea, and exaggerated emotional responses. This bidirectional network provides a mechanism of afferent and efferent feedback that may drive a migraine attack for many hours and even days (Burstein and Jakubowski, 2005).

As episodic migraine progresses to its chronic form, psychological dysfunction often increases concurrently (Smitherman et al., 2009). Patients with chronic migraine report more somatic complains including fatigue, sleep disturbances and nausea, compared to episodic migraineurs (Maizels and Burchette, 2004). Psychiatric comorbidity, including depression and anxiety, is also increased in chronic migraine (Buse et al., 2013). Migraine progression has been framed as a maladaptation to cumulative stress (allostatic load) over time, including the psychological stress associated with migraine both before and during attacks, as well as interictally (Borsook et al., 2012; Maleki et al., 2012a). In defining allostatic load as the amount of brain activity required to appropriately manage the level of emotional stress at any given time (McEwen and Gianaros, 2011), our results would suggest that even episodic migraineurs display an increased allostatic load in response to negative emotional stress. An avenue of further research would be to compare the response of episodic and chronic migraineurs to emotional stimuli, under the hypothesis that chronic migraineurs may display even greater brain activity as a marker of greater allostatic load. Interestingly, in the context of other pain conditions such as back pain, chronification has been demonstrated to involve a progressive shift away in pain processing from classical sensory regions and more toward engagement of emotional regions (Hashmi et al., 2013). In regards to migraine, determining if such a similar shift is involved in either the early development and/or transformation to chronicity would form a novel and important avenue of research in the evolution of migraine along the lifespan.

\section{Study Caveats and Future Considerations}

While this is the first study to demonstrate increased brain activity in response to general negative emotional stimuli in migraineurs, there are some caveats of our study that should be acknowledged. Though we have interpreted the observed increased activity in migraineurs in response to negative emotional stimuli as evidence of cortico-limbic hyperexcitability we are unable to determine if this represents increased facilitation or dishabituation of emotional processing networks (Coppola et al., 2007; Stankewitz and May, 2007). Additionally, although migraineurs and controls gave equal retrospective ratings of valence and arousal, migraineurs may have experience increased emotion or arousal during the novel presentation and/or reduced emotional habituation over the stimuli period, similar to that demonstrated in response to other stimuli (i.e., visual). By 
continuously rating during the scanning session, rather than retrospectively, this issue may be clarified in future studies. Additionally, future studies may also include psychophysiological measures, such as engagement of the autonomic nervous system, as additional measures of emotional perception (Bonnet et al., 2015). Furthermore, while emotional stimuli of varying valence (i.e., positive, neutral, and negative) were examined in the current study, future studies may also examine negative emotional stimuli with varying levels of arousal (i.e., high versus low) and salience (i.e., pain-related versus non-pain-related images) to determine if additional factors may also influence this increased central processing.

\section{CONCLUSION}

Migraine is a multifactorial disorder encompassing not only pain and sensory disturbances but also a complex psychological/affective component active both during attacks and interictaly. A complete model of migraine pathophysiology must integrate all of these diverse components that give rise to the condition (Burstein et al., 2015). Our findings of increased functional activity, namely in cortico-limbic areas including the PCC and amygdala in response to negative emotional stimuli in migraineurs, expands the idea of increased functional activity in response to sensory stimuli to also encompass negative affect. The repeated stress of each migraine attack, and the anticipation of such, results in an ongoing response to an aversive process. Over time, the load of repeated attacks may lead to a more generalized sensitivity to aversive stimuli. An overall enhanced response to negative/aversive stimuli in migraine may underlie the multi/cross sensitization observed in migraine. Equal focus on both the sensory and emotional components of migraine and its pathophysiology may promote a more unified model

\section{REFERENCES}

Aderjan, D., Stankewitz, A., and May, A. (2010). Neuronal mechanisms during repetitive trigemino-nociceptive stimulation in migraine patients. Pain 151, 97-103. doi: 10.1016/j.pain.2010.06.024

Aldhafeeri, F. M., Mackenzie, I., Kay, T., Alghamdi, J., and Sluming, V. (2012). Regional brain responses to pleasant and unpleasant IAPS pictures: different networks. Neurosci. Lett. 512, 94-98. doi: 10.1016/j.neulet.2012.01.064

Andreatta, M., Puschmann, A. K., Sommer, C., Weyers, P., Pauli, P., and Muhlberger, A. (2012). Altered processing of emotional stimuli in migraine: an event-related potential study. Cephalalgia 32, 1101-1108. doi: 10.1177/0333102412459573

Apkarian, A. V., Bushnell, M. C., Treede, R. D., and Zubieta, J. K. (2005). Human brain mechanisms of pain perception and regulation in health and disease. Eur. J. Pain 9, 463-484. doi: 10.1016/j.ejpain.2004.11.001

Ashkenazi, A., Mushtaq, A., Yang, I., and Oshinsky, M. L. (2009). Ictal and interictal phonophobia in migraine-a quantitative controlled study. Cephalalgia 29, 1042-1048. doi: 10.1111/j.1468-2982.2008.01834.x

Beck, A. T., Steer, R. A., Ball, R., and Ranieri, W. (1996). Comparison of beck depression inventories -IA and -II in psychiatric outpatients. J. Pers. Assess. 67, 588-597. doi: 10.1207/s15327752jpa6703_13

Bienvenu, T. C., Busti, D., and Micklem, B. R. (2015). Large intercalated neurons of amygdala relay noxious sensory information. J. Neurosci. 35, 2044-2057.

Bjork, M., Hagen, K., Stovner, L., and Sand, T. (2011). Photic EEGdriving responses related to ictal phases and trigger sensitivity in of migraine that may account for the diversity of triggers, the increased comorbidity with emotional disturbances and the progression from episodic to chronic migraine.

\section{AUTHOR CONTRIBUTIONS}

SW contributed to the analysis and interpretation of data, drafting of manuscript and critical revision; RV and SS contributed to the acquisition of data; JL contributed to the analysis of data; $\mathrm{DH}$ contributed to critical revision; $\mathrm{RB}$ contributed to study conception and design; LB contributed to study conception and design, analysis of data and critical revision and DB contributed to study conception and design and critical revision.

\section{FUNDING}

Research reported in this publication was supported by the National Institutes of Health (NIH) under award number R01 NS073977 and K24 NS064050.

\section{ACKNOWLEDGMENT}

We would like to thank the many participants who graciously volunteered to take part in this study.

\section{SUPPLEMENTARY MATERIAL}

The Supplementary Material for this article can be found online at: http://journal.frontiersin.org/article/10.3389/fnhum. 2016.00366

migraine: a longitudinal, controlled study. Cephalalgia 31, 444-455. doi: $10.1177 / 0333102410385582$

Bonnet, L., Comte, A., Tatu, L., Millot, J. L., Moulin, T., and de Bustos, E. (2015). The role of the amygdala in the perception of positive emotions: an "intensity detector”. Front. Behav. Neurosci. 9:178. doi: 10.3389/fnbeh.2015.00178

Borsook, D., Maleki, N., Becerra, L., and McEwen, B. (2012). Understanding migraine through the lens of maladaptive stress responses: a model disease of allostatic load. Neuron 73, 219-234. doi: 10.1016/j.neuron.2012.01.001

Boucher, O., D’Hondt, F., Tremblay, J., Lepore, F., Lassonde, M., Vannasing, P., et al. (2015). Spatiotemporal dynamics of affective picture processing revealed by intracranial high-gamma modulations. Hum. Brain mapp. 36, 16-28. doi: 10.1002/hbm.22609

Boulloche, N., Denuelle, M., Payoux, P., Fabre, N., Trotter, Y., and Geraud, G. (2010). Photophobia in migraine: an interictal PET study of cortical hyperexcitability and its modulation by pain. J. Neurol. Neurosurg. Psychiatry 81, 978-984. doi: 10.1136/jnnp.2009.190223

Bradley, M. M., and Lang, P. J. (1994). Measuring emotion: the self-assessment manikin and the semantic differential. J. Behav. Ther. Exp. Psychiatry 25, 49-59. doi: 10.1016/0005-7916(94)90063-9

Brighina, F., Palermo, A., and Fierro, B. (2009). Cortical inhibition and habituation to evoked potentials: relevance for pathophysiology of migraine. J. Headache pain 10, 77-84. doi: 10.1007/s10194-008-0095-x

Burstein, R., and Jakubowski, M. (2005). Unitary hypothesis for multiple triggers of the pain and strain of migraine. J. Comp. Neurol. 493, 9-14. doi: $10.1002 /$ cne. 20688 
Burstein, R., Jakubowski, M., Garcia-Nicas, E., Kainz, V., Bajwa, Z., Hargreaves, R., et al. (2010). Thalamic sensitization transforms localized pain into widespread allodynia. Ann. Neurol. 68, 81-91. doi: 10.1002/ana.21994

Burstein, R., Noseda, R., and Borsook, D. (2015). Migraine: multiple processes, complex pathophysiology. J. Neurosci. 35, 6619-6629. doi: 10.1523/JNEUROSCI.0373-15.2015

Buse, D. C., Silberstein, S. D., Manack, A. N., Papapetropoulos, S., and Lipton, R. B. (2013). Psychiatric comorbidities of episodic and chronic migraine. J. Neurol. 260, 1960-1969. doi: 10.1007/s00415-012-6725-x

Cauda, F., Torta, D. M., Sacco, K., Geda, E., D’Agata, F., Costa, T., et al. (2012). Shared "core" areas between the pain and other task-related networks. PLoS ONE 7:e41929. doi: 10.1371/journal.pone.0041929

Chavez, C. M., McGaugh, J. L., and Weinberger, N. M. (2009). The basolateral amygdala modulates specific sensory memory representations in the cerebral cortex. Neurobiol. Learn. Mem. 91, 382-392. doi: 10.1016/j.nlm.2008. 10.010

Coppola, G., Pierelli, F., and Schoenen, J. (2007). Is the cerebral cortex hyperexcitable or hyperresponsive in migraine? Cephalalgia 27, 1427-1439. doi: 10.1111/j.1468-2982.2007.01500.x

Cucchiara, B., Datta, R., Aguirre, G. K., Idoko, K. E., and Detre, J. (2015). Measurement of visual sensitivity in migraine: validation of two scales and correlation with visual cortex activation. Cephalalgia 35, 585-592.

DaSilva, A. F., Becerra, L., Makris, N., Strassman, A. M., Gonzalez, R. G., Geatrakis, N., et al. (2002). Somatotopic activation in the human trigeminal pain pathway. J. Neurosci. 22, 8183-8192.

de Tommaso, M., Calabrese, R., Vecchio, E., De Vito Francesco, V., Lancioni, G., and Livrea, P. (2009). Effects of affective pictures on pain sensitivity and cortical responses induced by laser stimuli in healthy subjects and migraine patients. Int. J. Psychophysiol. 74, 139-148. doi: 10.1016/j.ijpsycho.2009. 08.004

Demarquay, G., Royet, J. P., Giraud, P., Chazot, G., Valade, D., and Ryvlin, P. (2006). Rating of olfactory judgements in migraine patients. Cephalalgia 26, 1123-1130. doi: 10.1111/j.1468-2982.2006.01174.x

Demarquay, G., Royet, J. P., Mick, G., and Ryvlin, P. (2008). Olfactory hypersensitivity in migraineurs: a H(2)(15)O-PET study. Cephalalgia 28, 10691080. doi: 10.1111/j.1468-2982.2008.01672.x

Denuelle, M., Boulloche, N., Payoux, P., Fabre, N., Trotter, Y., and Geraud, G. (2011). A PET study of photophobia during spontaneous migraine attacks. Neurology 76, 213-218. doi: 10.1212/WNL.0b013e3182074a57

Desikan, R. S., Segonne, F., Fischl, B., Quinn, B. T., Dickerson, B. C., Blacker, D., et al. (2006). An automated labeling system for subdividing the human cerebral cortex on MRI scans into gyral based regions of interest. NeuroImage 31, 968-980. doi: 10.1016/j.neuroimage.2006.01.021

Duerden, E. G., and Albanese, M. C. (2013). Localization of pain-related brain activation: a meta-analysis of neuroimaging data. Hum. Brain Mapp. 34, 109 149. doi: $10.1002 / \mathrm{hbm} .21416$

Gao, Y. J., Ren, W. H., Zhang, Y. Q., and Zhao, Z. Q. (2004). Contributions of the anterior cingulate cortex and amygdala to pain- and fear-conditioned place avoidance in rats. Pain 110, 343-353. doi: 10.1016/j.pain.2004.04.030

Goadsby, P. J., Charbit, A. R., Andreou, A. P., Akerman, S., and Holland, P. R. (2009). Neurobiology of migraine. Neuroscience 161, 327-341. doi: 10.1016/j.neuroscience.2009.03.019

Gur, R. E., McGrath, C., Chan, R. M., Schroeder, L., Turner, T., Turetsky, B. I., et al. (2002). An fMRI study of facial emotion processing in patients with schizophrenia. Am. J. Psychiatry 159, 1992-1999. doi: 10.1176/appi.ajp.159.12.1992

Hadjikhani, N., Ward, N., Boshyan, J., Napadow, V., Maeda, Y., Truini, A., et al. (2013). The missing link: enhanced functional connectivity between amygdala and visceroceptive cortex in migraine. Cephalalgia 33, 1264-1268. doi: $10.1177 / 0333102413490344$

Harrigan, J. A., Kues, J. R., Ricks, D. F., and Smith, R. (1984). Moods that predict coming migraine headaches. Pain 20, 385-396. doi: 10.1016/03043959(84)90115-5

Hashmi, J. A., Baliki, M. N., Huang, L., Baria, A. T., Torbey, S., Hermann, K. M., et al. (2013). Shape shifting pain: chronification of back pain shifts brain representation from nociceptive to emotional circuits. Brain 136(Pt 9), 2751-2768. doi: 10.1093/brain/awt211
Hougaard, A., Amin, F. M., Magon, S., Sprenger, T., Rostrup, E., and Ashina, M. (2015). No abnormalities of intrinsic brain connectivity in the interictal phase of migraine with aura. Eur. J. Neurol. 22, 702-e746. doi: 10.1111/ene.12636

Iannetti, G. D., and Mouraux, A. (2010). From the neuromatrix to the pain matrix (and back). Exp. Brain Res. 205, 1-12. doi: 10.1007/s00221-010-2340-1

Kamping, S., Bomba, I. C., Kanske, P., Diesch, E., and Flor, H. (2013). Deficient modulation of pain by a positive emotional context in fibromyalgia patients. Pain 154, 1846-1855. doi: 10.1016/j.pain.2013.06.003

Kelman, L. (2007). The triggers or precipitants of the acute migraine attack. Cephalalgia 27, 394-402. doi: 10.1111/j.1468-2982.2007.01303.x

Lang, P. J., Bradley, M. M., and Cuthbert, B. N. (2008). International Affective Picture System (IAPS): Affective Ratings of Pictures and Instruction Manual. Technical Report A-8. Gainesville, FL: University of Florida.

Lanteri-Minet, M., Radat, F., Chautard, M. H., and Lucas, C. (2005). Anxiety and depression associated with migraine: influence on migraine subjects' disability and quality of life, and acute migraine management. Pain 118, 319-326. doi: 10.1016/j.pain.2005.09.010

Maizels, M., Aurora, S., and Heinricher, M. (2012). Beyond neurovascular: migraine as a dysfunctional neurolimbic pain network. Headache 52, 1553 1565. doi: 10.1111/j.1526-4610.2012.02209.x

Maizels, M., and Burchette, R. (2004). Somatic symptoms in headache patients: the influence of headache diagnosis, frequency, and comorbidity. Headache 44, 983-993. doi: 10.1111/j.1526-4610.2004.04192.x

Maleki, N., Becerra, L., and Borsook, D. (2012a). Migraine: maladaptive brain responses to stress. Headache 52(Suppl. 2), 102-106. doi: 10.1111/j.15264610.2012.02241.x

Maleki, N., Becerra, L., Brawn, J., Bigal, M., Burstein, R., and Borsook, D (2012b). Concurrent functional and structural cortical alterations in migraine. Cephalalgia 32, 607-620. doi: 10.1177/0333102412445622

Martin, H., del Rio, M., de Silanes, C. L., Alvarez-Linera, J., Hernandez, J. A., and Pareja, J. A. (2011). Photoreactivity of the occipital cortex measured by functional magnetic resonance imaging-blood oxygenation level dependent in migraine patients and healthy volunteers: pathophysiological implications. Headache 51, 1520-1528. doi: 10.1111/j.1526-4610.2011.02013.x

McEwen, B. S., and Gianaros, P. J. (2011). Stress- and allostasis-induced brain plasticity. Annu. Rev. Med. 62, 431-445. doi: 10.1146/annurev-med-052209100430

Meerwijk, E. L., Ford, J. M., and Weiss, S. J. (2013). Brain regions associated with psychological pain: implications for a neural network and its relationship to physical pain. Brain Imaging Behav. 7, 1-14. doi: 10.1007/s11682-0129179-y

Melzack, R. (1999). From the gate to the neuromatrix. Pain 82, S121-S126. doi: 10.1016/S0304-3959(99)00145-1

Merskey, H. (1968). Psychological aspects of pain. Postgrad. Med. J. 44, 297. doi: 10.1136/pgmj.44.510.297

Merskey, H., and Bogduk, N. (1994). Part III: Pain Terms, A Current List with Definitions and Notes on Usage. Classification of Chronic Pain, Seattle, WA: IASP Press.

Moulton, E. A., Becerra, L., Maleki, N., Pendse, G., Tully, S., Hargreaves, R., et al. (2011). Painful heat reveals hyperexcitability of the temporal pole in interictal and ictal migraine States. Cereb. Cortex 21, 435-448. doi: 10.1093/cercor/bhq109

Mulleners, W. M., Aurora, S. K., Chronicle, E. P., Stewart, R., Gopal, S., and Koehler, P. J. (2001). Self-reported photophobic symptoms in migraineurs and controls are reliable and predict diagnostic category accurately. Headache 41, 31-39. doi: 10.1046/j.1526-4610.2001.111006031.x

Neta, M., Kelley, W. M., and Whalen, P. J. (2013). Neural responses to ambiguity involve domain-general and domain-specific emotion processing systems. J. Cogn. Neurosci. 25, 547-557. doi: 10.1162/jocn_a_00363

Neugebauer, V. (2015). Amygdala pain mechanisms. Handb. Exp. Pharmacol. 227, 261-284. doi: 10.1007/978-3-662-46450-2_13

Peyron, R., Laurent, B., and Garcia-Larrea, L. (2000). Functional imaging of brain responses to pain. A review and meta-analysis (2000). Neurophysiol. clin 30, 263-288. doi: 10.1016/S0987-7053(00)00227-6

Phan, K. L., Wager, T., Taylor, S. F., and Liberzon, I. (2002). Functional neuroanatomy of emotion: a meta-analysis of emotion activation studies in PET and fMRI. NeuroImage 16, 331-348. doi: 10.1006/nimg.2002.1087 
Radat, F., and Swendsen, J. (2005). Psychiatric comorbidity in migraine: a review. Cephalalgia 25, 165-178. doi: 10.1111/j.1468-2982.2004.00839.x

Rojahn, J., and Gerhards, F. (1986). Subjective stress sensitivity and physiological responses to an aversive auditory stimulus in migraine and control subjects. J. Behav. Med. 9, 203-212. doi: 10.1007/BF00848478

Russo, A., Tessitore, A., Esposito, F., Marcuccio, L., Giordano, A., Conforti, R., et al. (2012). Pain processing in patients with migraine: an event-related fMRI study during trigeminal nociceptive stimulation. J. Neurol. 259, 1903-1912. doi: 10.1007/s00415-012-6438-1

Schwedt, T. J., Schlaggar, B. L., Mar, S., Nolan, T., Coalson, R. S., Nardos, B., et al. (2013). Atypical resting-state functional connectivity of affective pain regions in chronic migraine. Headache 53, 737-751. doi: 10.1111/head.12081

Shyu, B. C., and Vogt, B. A. (2009). Short-term synaptic plasticity in the nociceptive thalamic-anterior cingulate pathway. Mol. pain 5, 51. doi: 10.1186/1744-80695-51

Smitherman, T. A., Rains, J. C., and Penzien, D. B. (2009). Psychiatric comorbidities and migraine chronification. Curr. Pain Headache Rep. 13, 326331. doi: 10.1007/s11916-009-0052-7

Sorbi, M. J., Maassen, G. H., and Spierings, E. L. (1996). A time series analysis of daily hassles and mood changes in the 3 days before the migraine attack. Behav. Med. 22, 103-113. doi: 10.1080/08964289.1996.9933771

Spierings, E. L., Sorbi, M., Haimowitz, B. R., and Tellegen, B. (1996). Changes in daily hassles, mood, and sleep in the 2 days before a migraine headache. Clin. J. Pain 12, 38-42. doi: 10.1097/00002508-199603000-00007

Spierings, E. L., Sorbi, M., Maassen, G. H., and Honkoop, P. C. (1997). Psychophysical precedents of migraine in relation to the time of onset of the headache: the migraine time line. Headache 37, 217-220. doi: 10.1046/j.15264610.1997.3704217.x

Stankewitz, A., and May, A. (2007). Cortical excitability and migraine. Cephalalgia 27, 1454-1456. doi: 10.1111/j.1468-2982.2007.01503.x

Stankewitz, A., and May, A. (2011). Increased limbic and brainstem activity during migraine attacks following olfactory stimulation. Neurology 77, 476-482. doi: 10.1212/WNL.0b013e318227e4a8

Steppacher, I., Schindler, S., and Kissler, J. (2015). Higher, faster, worse? An eventrelated potentials study of affective picture processing in migraine. Cephalalgia 36, 249-257.
Vanagaite, J., Pareja, J. A., Storen, O., White, L. R., Sand, T., and Stovner, L. J. (1997). Light-induced discomfort and pain in migraine. Cephalalgia 17, 733741. doi: 10.1046/j.1468-2982.1997.1707733.x

Vingen, J. V., Pareja, J. A., Storen, O., White, L. R., and Stovner, L. J. (1998). Phonophobia in migraine. Cephalalgia 18, 243-249. doi: 10.1046/j.14682982.1998.1805243.x

Vogt, B. A. (2005). Pain and emotion interactions in subregions of the cingulate gyrus. Nat. Rev. Neurosci. 6, 533-544. doi: 10.1038/nrn1704

Walters, A. B., Hamer, J. D., and Smitherman, T. A. (2014). Sleep disturbance and affective comorbidity among episodic migraineurs. Headache 54, 116-124. doi: 10.1111/head.12168

Wiech, K., Jbabdi, S., Lin, C. S., Andersson, J., and Tracey, I. (2014). Differential structural and resting state connectivity between insular subdivisions and other pain-related brain regions. Pain 155, 2047-2055. doi: 10.1016/j.pain.2014.07.009

Wiech, K., and Tracey, I. (2009). The influence of negative emotions on pain: behavioral effects and neural mechanisms. Neuroimage 47, 987-994. doi: 10.1016/j.neuroimage.2009.05.059

Worsley, K. J. (2001). "Statistical analysis of activation images," in Functional MRI: An Introductionto Methods, eds P. Jezzard, P. M. Matthews, and S. M. Smith (New York, NY: Oxford University Press), 251-270.

Zald, D. H. (2003). The human amygdala and the emotional evaluation of sensory stimuli. Brain Res. Brain Res. Rev. 41, 88-123. doi: 10.1016/S01650173(02)00248-5

Conflict of Interest Statement: The authors declare that the research was conducted in the absence of any commercial or financial relationships that could be construed as a potential conflict of interest.

Copyright (C) 2016 Wilcox, Veggeberg, Lemme, Hodkinson, Scrivani, Burstein, Becerra and Borsook. This is an open-access article distributed under the terms of the Creative Commons Attribution License (CC BY). The use, distribution or reproduction in other forums is permitted, provided the original author(s) or licensor are credited and that the original publication in this journal is cited, in accordance with accepted academic practice. No use, distribution or reproduction is permitted which does not comply with these terms. 